\begin{tabular}{|c|l|}
\hline Title & Markov property and cokernels of local operators \\
\hline Author(s) & Iwata, K.; Schäfer, J. \\
\hline Citation & Hokkaido University Preprint Series in Mathematics, 259, 1-7 \\
\hline Issue Date & 199481 \\
\hline DOI & 10.14943/83406 \\
\hline Doc URL & http://hdl.handle.net/2115/69010 \\
\hline Type & bulletin (article) \\
\hline File Information & pre259.pdf \\
\hline
\end{tabular}

Instructions for use 


\section{MARKOV PROPERTY}

AND COKERNELS OF LOKAL OPERATORS

K. Iwata and J. Schäfer

Series $\sharp 259$. August 1994 


\section{HOKKAIDO UNIVERSITY \\ PREPRINT SERIES IN MATHEMATICS}

$\sharp 234$ : T. Nakazi, M. Yamada, Invertible Toeplitz operators and uniform algebras, 14 pages. 1994.

$\sharp 235$ : C. Dohmen, A Pohozaev-type inequality for a quasilinear Haraux-Weissler equation, 6 pages. 1994.

$\sharp 236$ : T. Mikami, Large deviations for the first exit time on small random perturbations of dynamical systems, 32 pages. 1994.

\$237 T. Ozawa, Characterization of Trudinger's inequality, 7 pages. 1994.

\#238 T. Hibi, Buchsbaum complexes with linear resolutions, 12 pages. 1994.

$\sharp 239$ Y. Giga, N. Mizoguchi, On time periodic solutions of the Dirichlet problem for degenerate parabolic equations of nondivergence type, 23 pages. 1994.

$\sharp 240$ C. Dohmen, Existence of Fast Decaying Solutions to a Haraux-Weissler Equation With a Prescribed Number of Zeroes, 12 pages. 1994.

$\sharp 241$ K. Sugano, Note on H-separable Frobenius extensions, 8 pages. 1994.

\#242 J. Zhai, Some Estimates For The Blowing up Solutions of Semilinear Heat Equations, 11 pages. 1994.

$\sharp 243$ N. Hayashi, K. Kato and T. Ozawa, Dilation Method and Smoothing Effect of the Schrödinger Evolution Group, 10 pages. 1994.

$\sharp 244$ D. Lehmann, T. Suwa, Residues of holomorphic vector fields relative to singular invariant subvarieties, 26 pages. 1994.

\#245 H. Kubo, Slowly decaying solutions for semilinear wave equations in odd space dimensions, 30 pages. 1994.

\#246 T. Nakazi, M. Yamada, ( $A_{2}$ )-Conditions and Carleson Inequalities, 27 pages. 1994.

$\sharp 247$ N. Hayashi, K. Kato and T. Ozawa, Dilation Method and smoothing Effect of Solutions to the Benjamin-ono Equation, 17 pages. 1994.

$\sharp 248$ H. Kikuchi, Sheaf cohcmology theory for measurable spaces, 12 pages. 1994.

$\sharp 249$ A. Inoue, Tauberian theorems for Fourier cosine transforms, 9 pages. 1994.

$\sharp 250$ S. Izumiya, G. T. Kossioris, Singularities for viscosity solutions of Hamilton-Jacobi equations, 23 pages. 1994.

$\sharp 251$ H. Kubo, K. Kubota, Asymptotic behaviors of radially symmetric solutions of $\square u=|u|^{p}$ for super critical values $p$ in odd space dimensions, 51 pages. 1994.

\#252 T. Mikami, Large Deviations and Central Limit Theorems for Eyraud-Farlie-Gumbel-Morgenstern Processes, 9 pages. 1994 .

\#253 T. Nishimori, Some remarks in a qualitative theory of similarity pseudogroups, 19 pages. 1994.

\#254 T. Suwa, Residues of complex analytic foliations relative to singular invariant subvarieties, 15 pages. 1994.

$\sharp 255$ T. Tsujishita, On Tripie Mutual Information, 7 pages. 1994.

$\sharp 256$ T. Tsujishita, Construction of Universal Modal World based on Hyperset Theory, 15 pages. 1994.

$\sharp 257$ A. Arai, Trace Formulas, a Golden-Thompson Inequality and Classical Limit in Boson Fock Space, 35 pages. 1994.

$\sharp 258$ Y-G. Chen, Y. Giga, T. Hitaka and M. Honma, A Stable Difference Scheme for Computing Motion of Level Surfaces by the Mean Curvature, 18 pages. 1994. 


\title{
MARKOV PROPERTY AND COKERNELS OF LOCAL OPERATORS
}

\author{
KOICHIRO IWATA AND JÖRG SCHÄFER
}

\begin{abstract}
We discuss Gaussian generalized random fields indexed by smooth sections of vector bundles with respect to Markov properties. We propose a new set-up which is suitable for the present question and within which new phenomena are detected naturally. In particular, we give a counterexample to the belief that locality in the RKHS implies germ Markov property. We also prove the close connection between Markov property and cokernels of local operators.
\end{abstract}

\section{INTRODUCTION}

In a paper by Preiss and Kotecky [8] delicate points concerning Markov properties of generalized random fields are discussed. They clearly pointed out the origin of confusions so far and they selected a presumably most suitable definition, which we shall also follow.

Let $\left\{X(\varphi) ; \varphi \in C_{0}^{\infty}\left(\mathbb{R}^{d}\right)\right\}$ be a generalized random field defined on a complete probability space $(\Omega, \mathcal{F}, P)$. If $D$ is an open subset of $\mathbb{R}^{d}$, then we define

$$
\mathcal{F}_{D}:=\sigma\{X(\varphi) ; \operatorname{supp} \varphi \subset D\} \vee \mathcal{N}
$$

where $\mathcal{N}$ is the trivial sub $\sigma$-field of $\mathcal{F}$. On the other hand we set

$$
\mathcal{F}_{C}:=\bigcap\left\{\mathcal{F}_{D} ; C \subset D, D \text { open }\right\}
$$

for closed subsets $C$. We shall use to denote the closure.

(1.1) Definition. We say that $\{X(\varphi)\}$ is germ Markov, if $\mathcal{F}_{\bar{D}} \perp \mathcal{F}_{\mathbb{R}^{d} \backslash D} \mid \mathcal{F}_{\partial D}$ for any open subset $D$. (Two sub $\sigma$-fields $\mathcal{F}_{\bar{D}}$ and $\mathcal{F}_{\mathbb{R}^{d} \backslash D}$ are conditionally independent given $\mathcal{F}_{\partial D}$.)

A superstition on Gaussian generalized random fields used to be that the germ Markov property is equivalent to the locality of the reproducing kernel Hilbert space for the covariance. A counterexample, given in [8], to the above assertion is a mean zero Gaussian system with

$$
E\left[X(\varphi)^{2}\right]=\int_{\mathbb{R}}\left\{\varphi(x)^{2}+\left(\varphi^{\prime}(x)\right)^{2}\right\} d x
$$

1991. Mathematics Subject Classification. Primary 60G20 Secondary 60G15, 60J99, 35B60.

The second author is grateful to the kind invitation to Hokkaido University where some of the present work was carried out. A subsequent visit of the first author at Ruhr-Universität Bochum stimulated the continuation of the joint work. 
We can check that $\mathcal{F}_{\bar{D}} \perp \mathcal{F}_{\mathbb{R} \backslash D}$ and $\mathcal{F}_{\partial D}=\mathcal{N}$ for any open subset by examining the corresponding condition in the Sobolev space carrying the inner product (1.2). Hence $\{X(\varphi)\}$ is germ Markov. But the bilinear form for the reproducing kernel Hilbert space (RKHS) has an integral kernel $\frac{1}{2} e^{-|x-y|}$ and therefore it is not local.

The superstition in former days was caused by the misunderstanding that the following would hold whenever open subsets $D_{+}, D_{-}$cover $\mathbb{R}^{d}$.

$$
\mathcal{F}_{D_{+}}=\mathcal{F}_{\mathbb{R}^{d} \backslash D_{-}} \vee \mathcal{F}_{D_{+} \cap D_{-}}
$$

However, as for the Gaussian system characterized by (1.2) we have $\mathcal{F}_{\mathbb{R}} \neq \mathcal{F}_{[0, \infty)} \vee$ $\mathcal{F}_{(-\infty, 0)}$, because the latter is independent of $\sigma\{X(f)\}$, where $f(x)=e^{-|x|}$. Thus this example also serves as a counterexample to (1.3).

Based on the discussion so far, Preiss and Kotecky [8] proposed the so-called "MI-Markov property" which is equivalent to the following definition.

(1.4) Definition. We say that $\{X(\varphi)\}$ is $c$-Markov, if it is germ Markov and satisfies

$$
\mathcal{F}_{\mathbb{R}^{d}}=\mathcal{F}_{\mathbb{R}^{d} \backslash D} \vee \mathcal{F}_{D} \text { for all open subsets } D \text { of } \mathbb{R}^{d}
$$

(1.6) Remark. We can prove that, actually, (1.4) is equivalent to the fact that $\mathcal{F}_{D_{1}} \perp \mathcal{F}_{D_{2}} \mid \mathcal{F}_{D_{1} \cap D_{2}}$ holds for any open covering $\left\{D_{1}, D_{2}\right\}$ of $\mathbb{R}^{d}$. This suggests the name "c"-Markov.

In order to compensate the incompleteness of this note concerning historical developments we refer to [3], [6], [11] and references therein. The present topic was also treated in the framework of Dirichlet spaces, see [5], [9] and [10]. However, within this framework one can only handle scalar random fields, i.e. fields indexed by scalar valued functions.

Therefore, in section 2 we introduce a new set-up which fits Gaussian generalized random fields indexed by sections of vector bundles and we explain the relation between a new Markov property to be defined below and the locality. Our new formulation can be proven to be equivalent to (1.4) as far as Gaussian systems are considered. In addition we shall mention the connection to the results mentioned above. Within our set-up degenerate fields, i.e. with degenerate covariance are easy to handle. Actually in the context of multicomponent fields very large degeneracy naturally happens, and in contrast to the non-degenerate scalar situation genuine new phenomena arise. This is shown in section 3, where we report on a Gaussian generalized random field whose reproducing kernel Hilbert space has locality but which is not germ Markov. Moreover, for a class of Gaussian generalized random fields we give a necessary and sufficient condition to be Markov (Theorem 3.13). This criterion is clearly different from those appearing in the context of Dirichlet spaces, see [10]. In particular, we see that locality of the RIHS is only part of the necessary condition. Together with the example of Preiss and Kotecky this shows the subtleties in the relation between germ Markov property and locality.

\section{THE MARKoV PROPERTY AND THE LOCALITY}

We formulate the question in terms of Hilbert space category, since we shall discuss Gaussian systems. Let $\mathbf{E} \rightarrow M$ be a real vector bundle over a paracompact $C^{\infty}$. manifold. We use the notation $\mathcal{D}=\mathcal{D}(\mathrm{E})$ for the totality of $C^{\infty}$-sections of $\mathrm{E}$ with 
compact supports. $\mathcal{D}$ is equipped with the Schwartz topology. Our objects to study are continuous linear maps

$$
\gamma: \mathcal{D} \longrightarrow \mathcal{H}
$$

with real Hilbert spaces $\mathcal{H}$ as targets, in which the images are dense. Given $\gamma: \mathcal{D} \longrightarrow \mathcal{H}$, we introduce a family of closed subspaces $\{\mathcal{H}(D)\}($ resp. $\{\mathcal{H}(C)\})$ of $\mathcal{H}$ indexed by open (resp. closed) subsets of $M$ as follows:

$$
\begin{aligned}
& \mathcal{H}(D):=\overline{\{\gamma(\varphi) ; \operatorname{supp} \varphi \subset D\}} \\
& \mathcal{H}(C):=\bigcap\{\mathcal{H}(D) ; C \subset D, D \text { open }\}
\end{aligned}
$$

In the study of a Gaussian generalized random field $\{X(\varphi) ; \varphi \in \mathcal{D}\}$, the map $\gamma: \mathcal{D} \longrightarrow \mathcal{H}$ is induced by the correspondence $\varphi \longmapsto X(\varphi)$ and therefore $\mathcal{H}$ is realized as a closed subspace of the $L^{2}$-space on $(\Omega, \mathcal{F}, P)$. We here adopt the conclusion of Lemma 3 from [3] as a definition of Markov property. In our context it reads as follows:

(2.2) Definition. The map $\gamma: \mathcal{D} \longrightarrow \mathcal{H}$ is called Markov if

$$
\mathcal{H}(\bar{D})=\mathcal{H}(\partial D)+\mathcal{H}(M \backslash D)^{\perp} \text { for all open subsets } D
$$

where $\perp$ means the orthogonal complement.

With the help of standard martingale convergence theorems and Wiener-Ito chaos decomposition one can prove that Definition 2.2 is actually another formulation of the $c$-Markov property for Gaussian generalized random fields. We recall the following fact, which is a standard tool to push the argument in the present subject and whose proof is thus omitted.

(2.3) Lemma. Let $\mathcal{K}_{0}, \mathcal{K}_{1}$ and $\mathcal{K}_{2}$ be closed subspaces of $\mathcal{H} . \mathcal{K}_{1}=\mathcal{K}_{0}+\left(\mathcal{K}_{1} \cap \mathcal{K}_{2}{ }^{\perp}\right)$ together with $\mathcal{K}_{0} \subset \mathcal{K}_{2}$ is equivalent to that $\mathcal{K}_{1} \cap \mathcal{K}_{2}=\mathcal{K}_{0}$ and $\mathcal{K}_{2}{ }^{\perp}=\left(\mathcal{K}_{1} \cap \mathcal{K}_{2}{ }^{\perp}\right)+$ $\left(\mathcal{K}_{1}+\mathcal{K}_{2}\right)^{\perp}$

The relation to the $c$-Markov property is now explained (with Remark 2.8 below taken into account).

(2.4) Theorem. The map $\gamma: \mathcal{D} \longrightarrow \mathcal{H}$ is Markov, if and only if

$$
\mathcal{H}=\overline{\mathcal{H}(\bar{D})+\mathcal{H}(M \backslash D)} \text { and } \mathcal{H}(\bar{D})=\mathcal{H}(\partial D)+\left(\mathcal{H}(\bar{D}) \cap \mathcal{H}(M \backslash D)^{\perp}\right)
$$

hold for all open subsets $D$.

(2.5) Remark. The latter condition appearing in (2.4) corresponds to the germ Markov property. It then follows from (2.3) that $\gamma: \mathcal{D} \longrightarrow \mathcal{H}$ is not germ Markov unless $\mathcal{H}(\partial D)=\mathcal{H}(\bar{D}) \cap \mathcal{H}(M \backslash D)$.

We see that the Markov property defined in (2.2) consists of two conditions: The first one reads as

$$
\mathcal{H}(M \backslash D)^{\perp} \subset \mathcal{H}(\bar{D})
$$


for all open subsets $D$. This condition has been formulated as locality of the dual (so called reproducing kernel) Hilbert space. In our set-up it is rather called semilocality. The second condition is given by

$$
\mathcal{H}(\partial D)=\mathcal{H}(\bar{D}) \cap \mathcal{H}(M \backslash D)
$$

for all open subsets $D$, which so far received less attention than (2.6). The reason is probably because within the framework of Dirichlet spaces (and scalar random fields) (2.7) is trivially fulfilled, compare [10]. However, within the framework of generalized random fields indexed by sections of bundles it appears very naturally as a necessary non-trivial condition.

(2.8) Remark. Since $\mathcal{H}=\mathcal{H}(M \backslash D)^{\perp}+\mathcal{H}(M \backslash D)$ is always valid, (2.6) implies that $\mathcal{H}=\mathcal{H}(\bar{D})+\mathcal{H}(M \backslash D)$. If (2.6) holds for all open subsets, then we can show that $\mathcal{H}=\mathcal{H}(D)+\mathcal{H}(M \backslash D)$. Therefore Definition 2.2 already includes the postulate corresponding to (1.5).

One of the aim in the present paper is to show by concrete examples that we really decompose Definition 2.2 into two independent parts. An example mentioned in the previous section is germ Markov and hence, according to Remark 2.5, satisfies (2.7), while the condition (2.6) breaks down. The other example we give, see Remark 3.12, satisfies (2.6), while the condition (2.7) breaks down. Thus, according to Remark 2.5 again, it is not germ Markov.

\section{A COUNTEREXAMPLE}

We mention a typical situation in which condition (2.7) fails. We suppose that $M$ is compact and connected. Given a fiber metric and a Radon measure chosen from the $C^{\infty}$-category, one can speak of square integrable sections of $\mathbf{E} \rightarrow M$. We will use the short hand notation $L^{2}(\mathrm{E})^{\prime}$ to denote the collection of elements in $\mathcal{D}^{\prime}(\mathrm{E})$ which are realizable as square integrable sections. Then clearly for each open subset $D$ we have the orthogonal direct sum decomposition

$$
\begin{aligned}
& \overline{\left\{f \in L^{2}(\mathrm{E})^{\prime} ; \operatorname{supp} f \subset M \backslash \partial D\right\}} \\
= & \overline{\left\{f \in L^{2}(\mathbf{E})^{\prime} ; \operatorname{supp} f \subset M \backslash \bar{D}\right\}} \oplus \overline{\left\{f \in L^{2}(\mathbf{E})^{\prime} ; \operatorname{supp} f \subset D\right\}} .
\end{aligned}
$$

If $L^{2}(\mathrm{E})^{\prime}$ in (3.1) is replaced by a subspace equipped with a stronger vector topology, condition (2.7) might break down, as we describe now using facts of the preceding paragraph. Let $\mathbf{F} \rightarrow M$ be another real vector bundle over $M$ and $L: \mathcal{D}(\mathbf{F}) \rightarrow \mathcal{D}(\mathrm{E})$ be a linear elliptic differential operator with transposed mapping $L^{\prime}: \mathcal{D}^{\prime}(\mathrm{E}) \rightarrow \mathcal{D}^{\prime}(\mathrm{F})$. We denote the cokernel $\mathcal{D}(\mathrm{E}) /$ Image $L$ by $\operatorname{coker} L$. Then coker $L$ and the kernel $\operatorname{ker} L^{\prime}$ are naturally paired by

$$
\operatorname{ker} L^{\prime}\langle h,[\varphi]\rangle_{\operatorname{coker} L}=\mathcal{D}^{\prime}\langle h, \varphi\rangle_{\mathcal{D}}, \quad h \in \operatorname{ker} L^{\prime}, \varphi \in \mathcal{D}(\mathrm{E}),
$$

where $\mathcal{D}(\mathbf{E}) \ni \varphi \mapsto[\varphi] \in \operatorname{coker} L$ is the canonical mapping. Since $L$ is a Fredholm operator according to the general theory of elliptic differential operators, see e.g. [1], this dualization determines the isomorphism $\operatorname{ker} L^{\prime} \simeq \operatorname{Hom}(\operatorname{coker} L, \mathbb{R})$ of two finite dimensional vector spaces.

Let $W^{\prime}$ be a subspace of $L^{2}(\mathrm{E})^{\prime}$ such that each $\varphi \in \mathcal{D}(\mathbf{E})$ whose support does not meet an open set $D$ is characterized by the property that $\mathcal{D}^{\prime}\langle f, \varphi\rangle_{\mathcal{D}}=0$ for all $f \in W^{\prime}$ with supp $f \subset D$. A typical example of such $W^{\prime}$ is a Sobolev space of positive order with an inner product obtained by usual coordinate patching. 
(3.2) Definition. Given an $L^{2}$-norm on $\mathcal{D}(\mathbf{E}), \Pi: W^{\prime} \rightarrow$ coker $L$ denotes the linear mapping characterized by

$$
\operatorname{ker} L^{\prime}\langle h, \Pi f\rangle_{\text {coker } L}=(h, f)_{L^{2}(\mathrm{E})^{\prime}} h \in \operatorname{ker} L^{\prime}, f \in W^{\prime} .
$$

Here we implicitly used the fact $\operatorname{ker} L^{\prime} \subset L^{2}(\mathrm{E})^{\prime}$, which follows from the elliptic regularity, see e.g. [1]. We intend to describe the so called unique continuation in terms of the mapping $\Pi$. We recall the following.

(3.3) Definition. We say $L^{\prime}$ has the unique continuation property if any $h \in \operatorname{ker} L^{\prime}$ vanishing on a non-empty open subset vanishes throughout $M$.

It is known that quite a large class of elliptic differential operators have the unique continuation property. We refer to [4] for this and the relation to strong continuation as well as for historical development.

(3.4) Lemma. The unique continuation property for $L^{\prime}$ with respect to a nonempty open subset $D$ is equivalent to the surjectivity of the mapping

$$
\left\{f \in W^{\prime} ; \operatorname{supp} f \subset D\right\} \ni f \longmapsto \Pi f \in \operatorname{coker} L .
$$

(3.5) Corollary. Let $\Pi$ be as in (3.2), and let $\mathcal{H}^{\prime}$ be a subspace of $W^{\prime}$ with ker $\Pi \subset \mathcal{H}^{\prime}$. Suppose that $\mathcal{H}^{\prime}$ is equipped with a vector topology such that the inclusion mapping $\mathcal{H}^{\prime} \rightarrow L^{2}(\mathrm{E})^{\prime}$ is continuous and moreover $L^{\prime}$ has the unique continuation property with respect to open subsets $D$ and $M \backslash \bar{D}$, both of which are non-empty. If

$$
\frac{\left\{v \in \mathcal{H}^{\prime} ; \operatorname{supp} v \subset M \backslash \partial D\right\}}{\subset\left\{v \in \mathcal{H}^{\prime} ; \operatorname{supp} v \subset M \backslash \bar{D}\right\}}+\overline{\left\{v \in \mathcal{H}^{\prime} ; \operatorname{supp} v \subset D\right\}}
$$

then $W^{\prime} \subset \mathcal{H}^{\prime}$

Proof. Given $f \in W^{\prime}$, with the help of Lemma 3.4, we can choose $f_{1}, f_{2} \in W^{\prime}$ so that $\operatorname{supp} f_{1} \subset M \backslash \bar{D}$ and $f_{1}+f \in \operatorname{ker} \Pi$, respectively $\operatorname{supp} f_{2} \subset D$ and $f_{2}-f \in \operatorname{ker} \Pi$. We set $v:=f_{1}+f_{2} \in \operatorname{ker} \Pi \subset \mathcal{H}^{\prime}$, which has the property that supp $v \subset M \backslash \partial D$. It then follows from (3.6) that there exist $v_{1}, v_{2} \in \mathcal{H}^{\prime}$ with $v_{1} \in \overline{\left\{v \in \mathcal{H}^{\prime} ; \operatorname{supp} v \subset M \backslash \bar{D}\right\}}$ and $v_{2} \in \overline{\left\{v \in \mathcal{H}^{\prime} ; \operatorname{supp} v \subset D\right\}}$ respectively, and $v=v_{1}+v_{2}$. Since the closure above was considered with respect to a vector topology stronger than the $L^{2}(\mathbf{E})^{\prime}$-topology, we see that $v_{1}$ is contained in the first term of the sum of the right-hand side of (3.1) and $v_{2}$ is contained in the second one. By virtue of (3.1), we must have $v_{1}=f_{1}$ and $v_{2}=f_{2}$, which implies $f_{1} \in \mathcal{H}^{\prime}$. Thus we get $f=f_{1}+f-f_{1} \in \operatorname{ker} \Pi+\mathcal{H}^{\prime} \subset \mathcal{H}^{\prime}$.

We now choose a Sobolev space of non-negative integer order as $W^{\prime}$.

(3.7) Lemma. Let $\mathcal{H}^{\prime}$ be a closed subspace of $W^{\prime}$. Then the following is equivalent to (3.6).

$$
\frac{\left\{v \in \mathcal{H}^{\prime} ; \operatorname{supp} v \subset M \backslash \partial D\right\}}{\subset\left\{v \in \mathcal{H}^{\prime} ; \operatorname{supp} v \subset M \backslash \bar{D}\right\}+\left\{v \in \mathcal{H}^{\prime} ; \operatorname{supp} v \subset D\right\}} .
$$


Proof. Clearly with respect to a natural Sobolev inner product we have

$$
\left\{v \in \mathcal{H}^{\prime} ; \operatorname{supp} v \subset M \backslash \bar{D}\right\} \perp\left\{v \in \mathcal{H}^{\prime} ; \operatorname{supp} v \subset D\right\} .
$$

Thus (3.8) is equivalent to (3.6).

We need a criterion whether a subspace $\mathcal{H}^{\prime}$ is closed in $W^{\prime}$. In the following lemma $W^{\prime}$ may be any subspace of $L^{2}(\mathrm{E})^{\prime}$.

(3.9) Lemma. Let $\Pi$ be as in (3.2), and let $\mathcal{H}^{\prime}$. be a subspace of $W^{\prime}$ with ker $\Pi \subset$ $\mathcal{H}^{\prime}$. Suppose that $W^{\prime}$ is equipped with a vector topology such that the inclusion mapping $W^{\prime} \rightarrow \mathcal{D}^{\prime}(\mathbf{E})$ is continuous. Then $\mathcal{H}^{\prime}$ is closed in $W^{\prime}$.

Proof. We set $N:=\left\{\Pi v \in \operatorname{coker} L ; v \in \mathcal{H}^{\prime}\right\}$. Since $\operatorname{ker} \Pi \subset \mathcal{H}^{\prime}$, we see that $\mathcal{H}^{\prime}=\left\{v \in W^{\prime} ; \Pi v \in N\right\}$. On the other hand the continuity of the inclusion mapping $W^{\prime} \rightarrow \mathcal{D}^{\prime}(\mathrm{E})$ implies that of $\Pi$, whose target is of finite dimension. Thus the statement follows.

We suppose that $L^{\prime}$ has the unique continuation property. For example we consider the Laplace operator for a compact Riemannian manifold. Let $\mathcal{H}^{\prime}$ be a subspace of $W^{\prime}$ with $\operatorname{ker} \Pi \subset \mathcal{H}^{\prime}$. Then, by Lemma $3.9, \mathcal{H}^{\prime}$ is closed in $W^{\prime}$. Any Hilbert space structure on $\mathcal{H}^{\prime}$ which forces the inclusion mapping $\mathcal{H}^{\prime} \rightarrow \mathcal{D}^{\prime}(\mathrm{E})$ continuous induces the same Fréchet topology as that induced by a natural Sobolev inner product on $W^{\prime}$, due to the closed graph theorem. Therefore all of the requirements in Corollary 3.5 and Lemma 3.7 are fulfilled. We denote the topological dual of $\mathcal{H}^{\prime}$ by $\mathcal{H}$. Since the dual pair $\mathcal{D}(\mathrm{E}), \mathcal{D}^{\prime}(\mathrm{E})$ is reflexive, a continuous linear mapping $\gamma: \mathcal{D}(\mathbf{E}) \longrightarrow \mathcal{H}$ is defined by

$$
\mathcal{H}^{\prime}\langle v, \gamma(\varphi)\rangle_{\mathcal{H}}=\mathcal{D}^{\prime}\langle v, \varphi\rangle_{\mathcal{D}}, \quad v \in \mathcal{H}^{\prime}, \varphi \in \mathcal{D}(\mathrm{E})
$$

We note that the image $\gamma(\mathcal{D})$ is dense in $\mathcal{H}$ and the canonical norm is given by

$$
\|\gamma(\varphi)\|_{\mathcal{H}}=\sup \left\{||_{\mathcal{D}^{\prime}}\langle v, \varphi\rangle_{\mathcal{D}} \mid ; v \in \mathcal{H}^{\prime},\|v\|_{\mathcal{H}^{\prime}} \leq 1\right\} \quad \varphi \in \mathcal{D}(\mathbf{E}) .
$$

Because (2.7) is the dual condition of (3.8) we get

(3.10) Proposition. Suppose $\mathcal{H}^{\prime} \neq W^{\prime}$. Then (2.7) breaks down for any non-void and non-dense open subset $D$ of $M$.

Let us adjust the order of the Sobolev space $W^{\prime}$ to the order of the differential operator $L$ and provide $W^{\prime}$ with an equivalent norm

$$
\|f\|_{W^{\prime}}:=\|\Pi f\|_{\operatorname{coker} L}+\left\|L^{\prime} f\right\|_{L^{2}(\mathrm{~F})^{\prime}} .
$$

Set $\mathcal{H}^{\prime}:=\left\{v \in W^{\prime} ; \Pi v=0\right\} .\|\cdot\|_{W^{\prime}}$ restricted on $\mathcal{H}^{\prime}$ is denoted by $\|\cdot\| \mathcal{H}^{\prime}$.

(3.12) Remark. $\|\cdot\|_{\mathcal{H}^{\prime}}$ is semi-local in the sense that two elements in $\mathcal{H}^{\prime}$ with disjoint supports are mutually orthogonal. Actually the semi-locality is the dual condition of (2.6). Therefore we get an example which satisfies (2.6) but does not satisfy (2.7) if coker $L \neq\{0\}$.

In order to describe the canonical norm for $\mathcal{H}$, we introduce the "Green" operator $G$ for $L$. Let $\Lambda: L^{2}(\mathrm{~F}) \rightarrow L^{2}(\mathrm{~F})$ denote the orthogonal projector with $\operatorname{ker} \Lambda=\operatorname{ker} L$. Then $G: \mathcal{D}(\mathbf{E}) \rightarrow \mathcal{D}(\mathbf{F})$ is determined by $G L \xi=\Lambda \xi$ for $\xi \in \mathcal{D}(\mathbf{F})$ and $\operatorname{ker} G=$ $(\text { Image } L)^{\perp}$. We now evaluate $\|\gamma(\varphi)\|_{\mathcal{H}}=\|G \varphi\|_{L^{2}(F)}$. This gives us at last the following theorem, cf. [11]. 
(3.13) Theorem. A mean 0 Gaussian random field $\{X(\varphi)\}_{\varphi \in \mathcal{D}}$ with $E\left[X(\varphi)^{2}\right]=$ $(G \varphi, G \varphi)_{L^{2}(\mathrm{~F})}$ is Markov iff $\operatorname{coker} L=\{0\}$.

(3.14) Remarks. (i) The theorem is inspired by solving $L^{\prime} X=Y$ for "Gaussian white noise" $Y$, see [2], [6] and [12].

(ii) In the theorem above we may replace "Markov", which actually means cMarkov, by any of the following Markovian properties: sharp Markov in the sense of Nelson, see [7], abstract Markov, see [12], or germ Markov.

In a separate article we shall present the logical development leading to the present example and the proofs in detail as well. Furthermore, we intend to prove, that (3.13) is the "generic" case in the sense that no matter how Markov property is interpreted there exist no Markovian solutions of stochastic PDE's described in Remark 3.14, unless $L$ has trivial cokernel.

\section{REFERENCES}

1. L. Hörmander, The Analysis of Linear Partial Differential Operators III, Springer, New York, 1983.

2. K. Iwata, The inverse of a local operator preserves the Markov property, Ann. Sc. Norm. Super. Pisa, Cl. Sci., IV. Ser. XIX (1992), 223-253.

3. G. Kallianpur, V. Mandrekar, The Markov property for generalized Gaussian random fields, Ann. Inst. Fourier 24 (1974), 143-167.

4. C. E. Kenig, Carleman Estimates, Uniform Sobolev Inequalities for Second Order Differential Operators, and Unique Continuation Theorems, Proc. of the Int. Cong. of Mathematicians, Berkeley, California, USA, 1986, pp. 948-960.

5. T. Kolsrud, On the Markov Property for Certain Gaussian Random Fields, Probab. Th. Rel. Fields 74 (1986), 393-402.

6. S. Kusuoka, Markov fields and local operators, J. Fac. Sci. Univ. Tokyo 1A (1979), 199-212.

7. E. Nelson, The construction of quantum fields from Markoff fields, J. Funct. Anal. 12 (1973), 97-112; The free Markoff field, J. Funct. Anal. 12 (1973), 211-227.

8. D. Preiss, R. Kotecky, Markov property of generalized random fields, Seventh Winter School, Czechoslovakia 1979.

9. M. Röckner, Markov Property of Generalized Fields and Axiomatic Potential Theory, Math. Ann. 264 (1983), 153-177.

10. M. Röckner, Generalized Markov Fields and Dirichlet Forms, Acta Appl. Math. 3 (1985), 285-311.

11. Yu. A. Rozanov, Markov Random Fields, Springer, 1982.

12. J. Schäfer, Abstract Markov Property and Local Operators, Bochum preprint (1994).

Department of Mathematics, Hokkaido University, Sapporo 060, Japan

E-mail address: iwata@math.hokudai.ac.jp

Fakultät für Mathematik, RUhr-Universität Bochum, 44780 Bochum, Germany

E-mail address: joerg.schaefer@ruba.rz.ruhr-uni-bochum.de 